\title{
On the Requirements for Successful GPS Spoofing Attacks
}

\author{
Nils Ole Tippenhauer \\ Dept. of Computer Science \\ ETH Zurich, Switzerland \\ tinils@inf.ethz.ch
}

\author{
Christina Pöpper \\ Dept. of Computer Science \\ ETH Zurich, Switzerland \\ poepperc@inf.ethz.ch
}

\author{
Kasper B. Rasmussen \\ Computer Science Dept. \\ UCl, Irvine, CA \\ kbrasmus@ics.uci.edu
}

\author{
Srdjan Čapkun \\ Dept. of Computer Science \\ ETH Zurich, Switzerland \\ capkuns@inf.ethz.ch
}

\begin{abstract}
An increasing number of wireless applications rely on GPS signals for localization, navigation, and time synchronization. However, civilian GPS signals are known to be susceptible to spoofing attacks which make GPS receivers in range believe that they reside at locations different than their real physical locations. In this paper, we investigate the requirements for successful GPS spoofing attacks on individuals and groups of victims with civilian or military GPS receivers. In particular, we are interested in identifying from which locations and with which precision the attacker needs to generate its signals in order to successfully spoof the receivers.

We will show, for example, that any number of receivers can easily be spoofed to one arbitrary location; however, the attacker is restricted to only few transmission locations when spoofing a group of receivers while preserving their constellation.

In addition, we investigate the practical aspects of a satellitelock takeover, in which a victim receives spoofed signals after first being locked on to legitimate GPS signals. Using a civilian GPS signal generator, we perform a set of experiments and find the minimal precision of the attacker's spoofing signals required for covert satellite-lock takeover.
\end{abstract}

\section{INTRODUCTION}

The Global Positioning System (GPS), originally introduced by the US military, has become an essential component for numerous civilian applications. Unlike military GPS signals, civilian GPS signals are not encrypted or authenticated and were never intended for safety- and security-critical applications. Nevertheless, GPSprovided locations are being used in applications such as vehicular navigation and aviation, asset monitoring (e.g., cargo tracking), and location-based services (e.g., routing) [22]. The use of the GPS system also includes time synchronization; examples are time stamping in security videos and critical time synchronization in financial, telecommunications and computer networks. Users highly rely on the precision and correctness of GPS location and time: transport companies depend on the correctness of localization to track trucks, cargoes, and goods under GPS surveillance, courts rely on criminals being correctly tracked by GPS-based an-

Permission to make digital or hard copies of all or part of this work for personal or classroom use is granted without fee provided that copies are not made or distributed for profit or commercial advantage and that copies bear this notice and the full citation on the first page. To copy otherwise, to republish, to post on servers or to redistribute to lists, requires prior specific permission and/or a fee.

Copyright 20XX ACM X-XXXXX-XX-X/XX/XX ...\$10.00. kle monitors, and aviation controls trust the correct monitoring of airplane traffic.

This heavy reliance on civilian GPS-following the discontinuation of the selective availability feature of GPS in the year 2000motivated a number of investigations on the security of GPS. These investigations found that civilian GPS is susceptible to jamming and spoofing attacks $[9,11,16,19]$. Successful spoofing experiments on standard receivers have been reported [7,23], showing that commercial-off-the-shelf receivers do not detect such attacks. The increased availability of programmable radio platforms such as USRPs [5] leads to a reduced cost of attacks on GPS. However, the requirements for GPS spoofing were so far not analyzed systematically and many of the previously proposed countermeasures $[8,16]$ assume a weak attacker that is, e.g., not able to generate signals with sufficient precision.

In this work, we investigate spoofing attacks on civilian and military GPS and analyze the requirements for their success as well as their limitations in practice. We divide the problem of GPS spoofing into the following two problems: (i) sending the correct spoofing signals such that they reach the receiver with the right timing, and (ii) getting a victim that is already synchronized to the legitimate GPS service to lock onto the attacker's spoofing signal. Regarding the first problem, we analyze the effects of GPS spoofing signals on multiple receivers and analyze under which conditions a group of victims can be spoofed such that, e. g., their mutual distances are preserved. Our analysis shows that, in order to spoof a group of victims while preserving the mutual distances, the attacker can only transmit from a restricted set of locations. To the best of our knowledge, such an analysis has not been done before. The second problem of taking over the satellite lock is relevant for performing attacks in real-world situations. In most cases, the victim will have been receiving legitimate GPS signals when the spoofing attack starts. It is thus important to know the required precision of the spoofing signal such that the victim seamlessly (i.e., without detection) switches lock from the legitimate GPS signal to the attacker's spoofing signal. We explore the influence of imperfections (in different aspects of signal power and timing) in a series of experiments and discuss the findings.

In short, our main contributions are as follows: First, we define the GPS group spoofing problem. Second, we analyze spoofing attacks on single and multiple receivers in civilian and military GPS systems and we infer theoretical bounds on the conditions for their success. Third, using a GPS signal generator ${ }^{1}$, we investigate the requirements for civilian GPS spoofing by seamless satellite-lock takeover under varying power, timing, and location precision of the attacker's spoofing signals and we provide bounds on these param-

\footnotetext{
${ }^{1}$ Satellite signal generators are also called satellite simulators-we use both notations in this paper.
} 
eters for the receiver used in our experiments.

The structure of the paper is as follows. We give background information on GPS positioning and discuss related work on GPS spoofing in Section 2. We introduce the GPS spoofing problem and our system and attacker models in Section 3. In Section 4, we analyze under which conditions GPS spoofing attacks are successful on single victims and groups of victims. The results of our experimental evaluation are presented in Section 5. In Section 6, we introduce a novel countermeasure against GPS spoofing attacks which is based on multiple receivers. We conclude the paper in Section 7.

\section{BACKGROUND}

In this section, we introduce the fundamental concepts of GPS (based on [11]) which are necessary for this work. We also summarize related work on the security of GPS.

\subsection{The Global Positioning System}

The Global Positioning System (GPS) uses a number of satellite transmitters $S_{i}$ located at known locations $L_{i}^{S} \in \mathbb{R}^{3}$. Each transmitter is equipped with a synchronized clock with no clock offset to the exact system time $t^{S}$ and broadcasts a carefully chosen navigation signal $s_{i}(t)$ (low auto-/cross-correlation ${ }^{2}$, including timestamps and information on the satellites' deviation from the predicted trajectories). The signal propagates with speed $c$ (see Figure 1).

A receiver $V$ located at the coordinates $L \in \mathbb{R}^{3}$ (to be determined) and using an omnidirectional antenna will receive the combined signal of all satellites in range:

$$
g(L, t)=\sum_{i} A_{i} s_{i}\left(t-\frac{\left|L_{i}^{S}-L\right|}{c}\right)+n(L, t)
$$

where $A_{i}$ is the attenuation that the signal suffers on its way from $L_{i}^{S}$ to $L,\left|L_{i}^{S}-L\right|$ denotes the Euclidean distance between $L_{i}^{S}$ and $L$, and $n(L, t)$ is background noise.

Due to the properties of the signals $s_{i}(t)$, the receiver can separate the individual terms of this sum and extract the relative spreading code phase, satellite ID, and data content using a replica of the used spreading code. Given the data and relative phase offsets, the receiver can identify the time delay $\left|L_{i}^{S}-L\right| / c$ for each satellite and from that infer the "ranges"

$$
d_{i}=\left|L_{i}^{S}-L\right| .
$$

With three known ranges $d_{i}$ to known transmitter positions $L_{i}^{S}$, three equations (2) can be solved unambiguously for $L$ (unless all three $S_{i}$ are located on a line). Since highly stable clocks (e.g., cesium oscillators) are costly and GPS receivers cannot participate in two-way clock synchronization, in practice, $V$ will have a clock offset $\delta$ to the exact system time: $t=t^{S}+\delta$. With this, Eq. 1 can be rewritten:

$$
g\left(L, t^{S}\right)=\sum_{i} A_{i} s_{i}\left(t-\frac{d_{i}}{c}-\delta\right)+n\left(L, t^{S}\right)
$$

where the receiver can only infer the "pseudoranges" $R_{i}$ from the delays $d_{i} / c+\delta$ :

$$
R_{i}=d_{i}+c \cdot \delta
$$

\footnotetext{
${ }^{2}$ In civilian GPS, the signals are spread using publicly known spreading codes. The codes used for military GPS are kept secret; they serve for signal hiding and authentication.
}

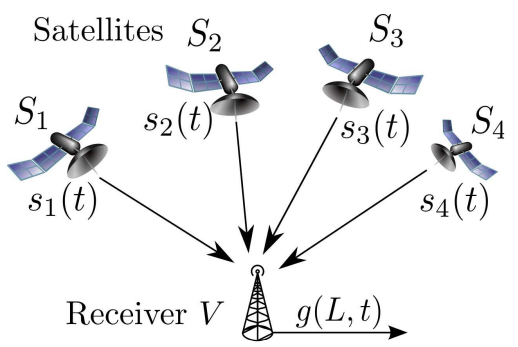

Figure 1: A GPS receiver $V$ works by observing the signals from a set of satellites. The relative delays of the signals $s_{i}(t)$ can be used to solve four equations which determine the 3dimensional position $L$ and the time offset $\delta$ of the receiver $V$.

The clock offset $\delta$ adds a fourth unknown scalar. With pseudorange measurements to at least four transmitters $S_{i}$, the resulting system of equations (4) can be solved for both $L$ and $\delta$, providing both the exact position and time, without requiring a precise local clock. Given $L_{i}^{S}=\left(x_{i}^{S}, y_{i}^{S}, z_{i}^{S}\right), L=(x, y, z)$, and $\Delta=c \cdot \delta$, we can transform (4) into the following set of equations [1]:

$$
\left(x-x_{i}^{S}\right)^{2}+\left(y-y_{i}^{S}\right)^{2}+\left(z-z_{i}^{S}\right)^{2}=\left(R_{i}-\Delta\right)^{2} \quad \forall S_{i}
$$

Geometrically, given a $\Delta$, each $S_{i}$ 's equation translates into a sphere with $L_{i}^{S}$ being the center. The set of equations (5) is overdetermined for more than four satellites and generally does not have a unique solution for $L$ because of data noise. It can be solved by numerical methods such as a least-mean-square approach or Newton's method [1]

\subsection{Related Work}

In 2001, the Volpe report [8] identified that (malicious) interference with the civilian GPS signal is a serious problem. Starting with this report, practical spoofing attacks were discussed in several publications. In [23], the authors use a WelNavigate GS720 satellite simulator mounted in a truck to attack a target receiver in a second truck. The authors succeeded in taking over the victim's satellite lock by manually placing an antenna close to the victim's receiver. After the victim was locked onto the attacker's signal the spoofing signal could be sent from a larger distance. Instead of using a GPS simulator, the authors of [7] create GPS spoofing signals by decoding legitimate GPS signals and generating timeshifted copies which are then transmitted with higher energy to overshadow the original signals; a similar approach is also used in [14]. This approach requires less expensive equipment but introduces considerable delays between the legitimate and the spoofed signals. GPS spoofing attacks are discussed analytically in [11], showing that an attacker can manipulate the arrival times of military and civilian GPS signals by pulse-delaying or replaying (individual) navigation signals with a delay. We note that there is no unique attacker model used for spoofing attacks, and thus the assumptions on the attacker's capabilities vary between these works.

Given the lack of attacker models, the proposed countermeasures range from simple measures to constant monitoring of the channel. In [8], consistency checks based on inertial sensors, cryptographic authentication, and discrimination based on signal strength, timeof-arrival, polarization, and angle-of-arrival are proposed. The authors of $[16,17,24]$ propose countermeasures based on detecting the side effects of a (not seamless) hostile satellite-lock takeover, e.g., by monitoring the local clock and Doppler shift of the signals. Kuhn proposes an asymmetric scheme in [11], based on the delayed disclosure of the spreading code and timing information. 


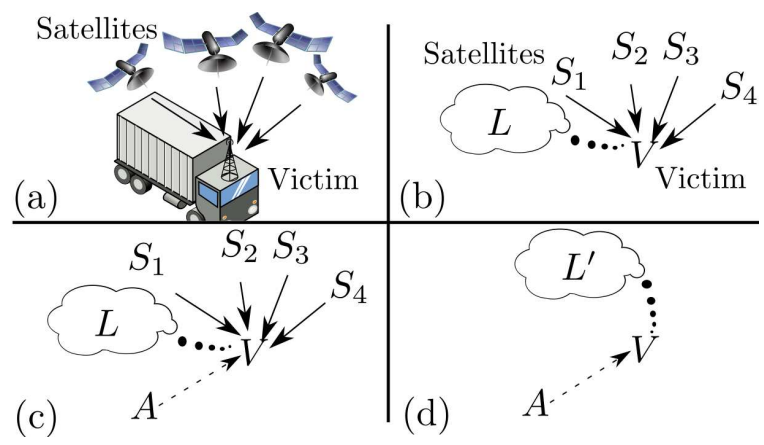

Figure 2: Basic attack scenario. (a) Visualization of the setup. The victim uses a GPS-based localization system and is synchronized to the legitimate satellites. (b) Abstract representation of the scene. (c) The attacker starts sending own spoofing and jamming signals. (d) The victim synchronizes to the attacker's signals.

In general, countermeasures that rely on modifications of the GPS satellite signals or the infrastructure (such as [11] and certain proposals in [8]) are unlikely to be implemented in the near future due to long procurement and deployment cycles. At the same time, countermeasures based on lock interrupts or signal jumps do not detect seamless satellite-lock takeovers.

Few publications $[3,12-14]$ present experimental data on the effects seen by the victim during a spoofing attack. The authors of [13] use a setup based on two antennas to measure the phase difference for each satellite to detect the lock takeover. [3] and [14] analyze the spoofing effect on the carrier and code level. The authors of [12] present a device that prevents spoofing by monitoring and potentially suppressing the received signals before they are processed by the GPS receiver.

All works above only consider attacks on single GPS receivers but not on groups of receivers. In addition, none of them investigated the requirements for successful attacks on public GPS receivers, such as required precision of the attacker's spoofing signals. Although we expect that more works on GPS spoofing and anti-spoofing countermeasures were performed in classified (military) settings, they are not accessible to the public.

\section{PROBLEM FORMULATION}

In order to give an intuition of the problem, we present our motivation and an exemplary use case. Subsequently we define our system and attacker models and formulate the GPS spoofing problem.

\subsection{Motivation}

The fundamental reasons why GPS spoofing works have been discussed in the literature before, and spoofing attacks have been demonstrated on single receivers experimentally. In this work, we show under which conditions the attacker can establish the correct parameters to launch a successful spoofing attack on one or more victims, and later in the experiments, how inaccuracies in these parameters influence the lock takeover during the attack. This analysis enables us to identify which attacks are theoretically possible and which attacks would be noticeable as (potentially nonmalicious) signal loss at the GPS receivers. This is important for proposing effective receiver-based countermeasures, which are not implemented yet in current standard GPS receivers.

Our work is further motivated by the real-life spoofing attacks,

\begin{tabular}{llll}
\hline$S_{i}$ & $i$-th satellite & $A_{i}$ & $i$-th attacker unit \\
$L_{i}^{S}$ & coordinates of $S_{i}$ & $P_{i}^{A}$ & physical coordinates of $A_{i}$ \\
$s_{i}$ & signal sent by $S_{i}$ & $L_{i}^{A}$ & claimed coordinates of $A_{i}$ \\
$V_{j}$ & $j$-th victim (receiver) & $s_{i}^{A}$ & signal sent by $A_{i}$ \\
$L_{j}$ & GPS coordinates of $V_{j}$ & $\delta_{i}^{A}$ & time offset of $s_{i}^{A}$ \\
$L_{j}^{\prime}$ & spoofed coordinates of $V_{j}$ & $\delta_{j}$ & GPS clock offset of $V_{j}$ \\
$P_{j}$ & physical coordinates of $V_{j}$ & $\delta_{j}^{\prime}$ & spoofed clock offset of $V_{j}$ \\
$R_{i j}^{\prime}$ & $V_{j}$ 's calculated PR to $S_{i}$ & $c$ & signal propagation speed \\
$R_{i j}^{A}$ & $V_{j}$ 's spoofed PR (by $A_{i}$ ) & $\Delta_{j}^{\prime}$ & $=\delta_{j}^{\prime} \cdot c$ \\
\hline
\end{tabular}

Table 1: Summary of notations (PR = pseudorange).

e.g. the one reported in [23]. In this scenario, a cargo truck (the victim), had a GPS unit that was housed in a tamper-proof casing and was sending cryptographically authenticated status updates with a fixed rate to a monitoring center. The attacker planned to steal the truck to get access to its loaded goods at a remote place. He got close to the victim and started transmitting forged (spoofed) signals in order to modify the location computed by the receiver (see Figure 2). In this setting, if the attacker can influence the localization process, he can make the victim report positions to the monitoring center that are unrelated to its actual physical position and thus steal the truck without raising suspicion or revealing the truck's real location.

\subsection{System Model}

Our system consists of a set of legitimate GPS satellites and a set $\mathcal{V}$ of victims (see Table 1 for notations used). Each victim is equipped with a GPS receiver that can compute the current position and time as described in Section 2. We assume that each receiver $V_{j} \in \mathcal{V}$ is able to receive wireless GPS signals, compute its position, and store its position/time-tuples. If several GPS receivers belong to a common group (e.g., they are mounted on the same vehicle), we assume that they can communicate to exchange their computed locations or are aware of the group's (fixed) formation.

The GPS location of each individual victim $V_{j} \in \mathcal{V}$ is given by its coordinates $L_{j} \in \mathbb{R}^{3}$ in space and the victim's clock offset $\delta_{j}$ with respect to the GPS system time $t^{S}$. We note that the computed GPS coordinates $L_{j}$ and clock offset $\delta_{j}$ do not necessarily correspond to the true (physical) coordinates $P_{j} \in \mathbb{R}^{3}$ and time. ${ }^{3}$ We define the local time of $V_{j}$ as $t_{j}=t^{S}+\delta_{j}$, i. e., $\delta_{j}<0$ refers to an internal clock that lags behind. We use $\mathcal{L}$ to denote the set of GPS locations of the victims in $\mathcal{V}$.

A GPS spoofing attack may manipulate a receiver's coordinates in space and/or its local time. We denote a victim's spoofed coordinates by $L_{j}^{\prime} \in \mathbb{R}^{3}$ and the spoofed time offset by $\delta_{j}^{\prime}$. We use $\mathcal{L}^{\prime}$ for the set of spoofed victim locations.

In our analysis in Section 4, we distinguish between civilian $G P S$, which uses the public C/A codes so that each satellite signal $s_{i}$ contains only public information, and military GPS, which provides authentic, confidential signals using the secret $\mathrm{P}(\mathrm{Y})$ codes. In the experimental evaluation in Section 5, we use a satellite signal generator for civilian GPS.

\subsection{Attacker Model}

GPS signals can be trivially spoofed under a Dolev-Yao [4]-like attacker that is able to fully control the wireless traffic by intercepting, injecting, modifying, replaying, delaying, and blocking messages without temporal constraints for individual receivers, see Figure 3(b). If the attacker has full control over the input to each individual receiver antenna, he can send the signals as they would $\overline{{ }^{3} \text { Typically, the difference }|L-P|}$ is less than a few meters [21]. 

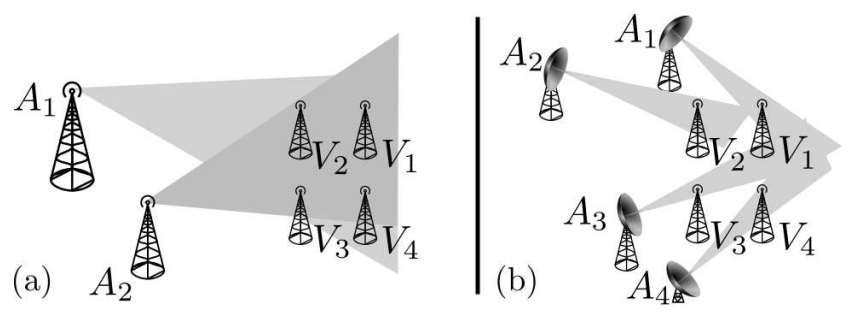

Figure 3: Models of the attacker's antenna coverage. (a) The attacker's signals reach all victims (used in the analysis of this paper). (b) The attacker's antennas each only reach one victim. This requires the attacker to be in close proximity to the victims if the distances between the receivers are small.

be received at any location $L_{j}^{\prime}$. This would, however, require the attacker to either be very close to each receiver or to use directional antennas with narrow beam widths and shielding to prevent that the signals intended for one victim are also received by another victim; in both cases, the number of required attacker antennas would be linear in the number of

victims. In this work we

assume that the signals sent by the attacker are transmitted wirelessly and that they will be received by all victims in $\mathcal{V}$, see Figure 3(a).

The attacker controls a set of wireless transmitters that he can move and position independently. We denote by $P_{i}^{A} \in \mathbb{R}^{3}$ the physical location of the $i$-th transmission unit of the attacker (manipulating the signals of satellite $S_{i}$ ), and the set of all physical attacker locations from where the attacker is transmitting by $\mathcal{P}^{A}$. We assume that the attacker's inherent, unwanted clock offset to the GPS system time is negligible ${ }^{4}$ and use $\delta_{i}^{A}$ to capture the time shift introduced by the attacker in the transmission of signal $s_{i}^{A}$ with respect to the signal $s_{i}$ and the system time $t^{S}$. For example, for $\delta_{1}^{A}=10 \mathrm{~ms}$, the attacker transmits the spoofed signal $10 \mathrm{~ms}$ after the signal $s_{1}$ was transmitted by satellite $S_{1}$.

For our analysis, we assume that the attacker is aware of the victims' physical locations (the influence of errors in the attackers location estimates is evaluated in Section 5). We further denote by $\left|L_{i}^{S}-P_{j}\right|$ the physical distance between satellite $S_{i}$ and victim $V_{j}$. Similarly, $\left|P_{i}^{A}-P_{j}\right|$ denotes the physical distance between the attacker's antenna at $P_{i}^{A}$ and victim $V_{j}$. Given this setting, we distinguish the following two types of attacks:

Attacks on civilian (unauthenticated) GPS: The attacker can delay signals or send them prematurely, i. e., $\delta_{i}^{A} \in \mathbb{R}$. He can modify the content of received GPS signals or arbitrarily generate the spoofing signals $s_{i}^{A}$ using the public GPS parameters (e. g., by using a GPS signal generator). This is possible because civilian GPS signals are not authenticated-given the right hardware, anyone can transmit his own GPS signals. Thus the attacker can also modify the claimed locations of the satellites: $L_{i}^{A} \neq L_{i}^{S}$. We note that on standard GPS receivers, the data content in the received GPS signals is not checked for plausibility or consistency [15].

Attacks on military (authenticated) GPS: The attacker is not able to generate valid military GPS signals. All he can do is to capture and relay existing signals, e. g. by separating signals from different satellites using high-gain directional antennas and broadband transceivers (called Selective-Delay in [11]). This means that the attacker can delay existing GPS signals and amplify or attenuate them. He is restricted by $\delta_{i}^{A} \geq$

\footnotetext{
${ }^{4}$ The attacker can synchronize his time to legitimate GPS signals.
}

$\left|L_{i}^{S}-P_{i}^{A}\right| \cdot c$, i. e., signals can be delayed but not sent prior to their reception. We note that neither the spreading codes nor the data content of the signal need to be known to the attacker for a successful selective-delay attack.

We note that these attacker models are very strong. Nevertheless, we consider them appropriate for our analysis because we want to make general statements that hold even under very strong (worstcase) attackers with sophisticated equipment.

\subsection{Formulation of GPS Spoofing Problems}

We first define GPS spoofing attacks and then present two GPS spoofing problems for the attacker.

Definition 1 (GPS Spoofing Attack). Let a victim $V$ compute its GPS location as L and its GPS time as $t$ in the absence of an attacker. In a GPS spoofing attack, the attacker sends spoofing signals to manipulate the victim's GPS-based location calculations. As a result, $V$ computes its location as $L^{\prime} \neq L$ and/or time as $t^{\prime} \neq t$.

Definition 1 can also be extended to groups of victims:

Definition 2 (GPS Group Spoofing Problem). Let $\mathcal{L}^{\prime}$ be a set of target locations for each $V_{j} \in \mathcal{V}$ and let $t_{j}^{\prime} \in \mathcal{T}^{\prime}$ denote the target time for $V_{j}$. The GPS Group Spoofing Problem is the problem of finding combinations of GPS signals $s_{i}^{A}$ (sent by the attacker), transmission times $t_{i}^{A}=t^{S}+\delta_{i}^{A}$ (when the spoofing signals are sent), and physical transmission locations $P_{i}^{A}$ (from where the attacker transmits) such that the location or time of each $V_{j} \in \mathcal{V}$ is spoofed according to Definition 1.

We note that the physical attacker locations $P_{i}^{A}$ do not have to correspond to the claimed satellite positions $L_{i}^{A}$ in the GPS messages (for civilian GPS, $L_{i}^{A}$ can even be chosen by the attacker). As we will show in Section 4.2, the GPS spoofing problem for a single victim has a trivial solution for any target location.

In Section 4.3, we will analyze the necessary restrictions on the spoofed locations such that the GPS Group Spoofing Problem can be solved. We therefore define a decisional version of the GPS Group Spoofing Problem.

Definition 3 (Decisional GPS Group Spoofing Problem). Let $\mathcal{P}$ be the set of physical locations of the victims in $\mathcal{V}$. Let $\mathcal{L}^{\prime}$ and $\mathcal{T}^{\prime}$ be defined according to Definition 2. The Decisional GPS Group Spoofing Problem for $\mathcal{P}, \mathcal{L}^{\prime}, \mathcal{T}^{\prime}$ is the decision problem whether there exists at least one set of attacker locations $\mathcal{P}^{A}$ from where the attacker can send the spoofing signals $s_{i}^{A}$ such that the location or time of each victim $V_{j} \in \mathcal{V}$ is spoofed according to Definition 1.

In practice, the GPS Group Spoofing Problems (Definitions 2 and 3) may be restricted in terms of attacker capabilities. For example, the attacker may only be able to position his transmission antennas at a restricted set of physical locations $\mathcal{P}_{*}^{A}$, at a restricted set of claimed satellite positions $\mathcal{L}_{*}^{A}$, or he may only be able to send the spoofing signals at a restricted set of transmission times $\mathcal{T}_{*}^{A}$ (e. g., if he must receive legitimate signals before he can send the spoofing signals). In these cases, the GPS Group Spoofing Problems can be modified to take the restricted attacker capabilities $\mathcal{L}_{*}^{A}, \mathcal{P}_{*}^{A}, \mathcal{T}_{*}^{A}$ as additional input and find solutions that fulfill $\mathcal{P}^{A} \subset \mathcal{P}_{*}^{A}, \mathcal{L}^{A} \subset \mathcal{L}_{*}^{A}$, or $\mathcal{T}^{A} \subset \mathcal{T}_{*}^{A}$.

\section{SOLVING GPS SPOOFING PROBLEMS}

We now analyze how our attacker (as defined in Section 3.3) can spoof the locations of one or more receivers. In this section, we abstract away from implementation issues (such as taking over an established lock to legitimate satellites, see Section 5), and assume that there are no legitimate signals present on the channel. 

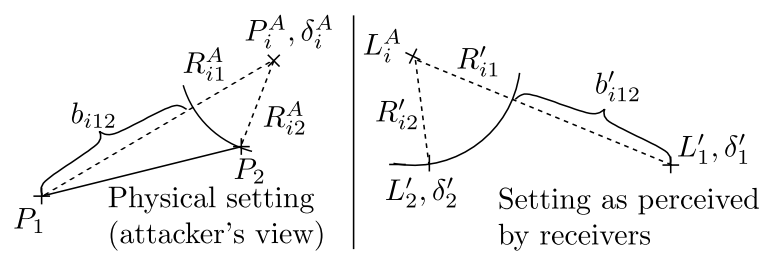

Figure 4: The GPS spoofing scenario for two victims in 2 dimensions. The attacker is impersonating a satellite with the claimed (forged) location $L_{i}^{A}$, using an antenna positioned at $P_{i}^{A}$. The victims are two receivers with physical positions at $P_{1}$ and $P_{2}$. For each signal $s_{i}^{A}$, the attacker ensures that $R_{i 1}^{A}$ and $R_{i 2}^{A}$ match $R_{i 1}^{\prime}$ and $R_{i 2}^{\prime}$, and therefore $V_{1}$ and $V_{2}$ compute their locations as $L_{1}^{\prime}$ and $L_{2}^{\prime}$ with clock offsets $\delta_{1}^{\prime}$ and $\delta_{2}^{\prime}$. Here, $b_{i 12}$ and $b_{i 12}^{\prime}$ are the differences of pseudoranges between $V_{1}$ and $V_{2}$.

\subsection{Construction of Pseudoranges}

The attacker's physical location $P_{i}^{A}$, his transmission time offset $\delta_{i}^{A}$, and the claimed satellite position $L_{i}^{A}$ all influence the location $L_{j}^{\prime}$ as computed by a victim $V_{j}$ (see Sections 2 and 3.2). By setting his physical location $P_{i}^{A}$ and transmission offset $\delta_{i}^{A}$, the attacker can influence the pseudorange computation at the victim. The expected pseudorange that a victim at physical position $P_{j}$ will compute based on the attacker's signal $s_{i}^{A}$ is

$$
R_{i j}^{A}=\left|P_{j}-P_{i}^{A}\right|+\delta_{i}^{A} \cdot c
$$

To determine its location, each victim solves a system of equations with the calculated pseudoranges (see Figure 4):

$$
\left|L_{j}^{\prime}-L_{i}^{A}\right|=R_{i j}^{\prime}-\Delta_{j}^{\prime}
$$

Here, $L_{i}^{A}$ are the (claimed) satellite coordinates of $S_{i}$ extracted by $V_{j}$ from the GPS message, $R_{i j}^{\prime}$ is the pseudorange to satellite $S_{i}$ as calculated by $V_{j}$ based on the received signal, and $\Delta_{j}^{\prime}=\delta_{j}^{\prime} \cdot c$ is the time offset times propagation speed as calculated by the victim.

For each impersonated satellite, the attacker must send a signal $s_{i}^{A}$ such that solving Equation 7 by the victim yields the target location $L_{j}^{\prime}$ and the target time offset $\delta_{j}^{\prime}$. This requires $R_{i j}^{A}=R_{i j}^{\prime}$, or:

$$
\left|P_{j}-P_{i}^{A}\right|+\Delta_{i}^{A}=\left|L_{j}^{\prime}-L_{i}^{A}\right|+\Delta_{j}^{\prime} .
$$

In attacks on civilian GPS, the attacker is free to choose $P_{i}^{A}$, $\delta_{i}^{A}$, and $L_{i}^{A}$. This means that the system of equations (8) is underdetermined for a single victim. The attacker can fix two of the variables to his liking and solve for the third.

When the attack targets a military GPS receiver, the attacker cannot change the data content of the messages and is restricted to $\delta_{i}^{A}$, which is greater than or equal to the transmission delay from the satellite to the attacker. Hence, the claimed satellite location in the message is the correct location of the legitimate satellite: $L_{i}^{A}=$ $L_{i}^{S}$. In addition, the attacker is restricted by $\Delta_{i}^{A} \geq\left|P_{i}^{A}-L_{i}^{S}\right|$. We can therefore rewrite Equation 8 as

$$
\left|P_{j}-P_{i}^{A}\right|+\left|P_{i}^{A}-L_{i}^{S}\right| \leq\left|L_{j}^{\prime}-L_{i}^{A}\right|+\Delta_{j}^{\prime} .
$$

Or, using the triangle inequality

$$
\left|P_{j}-L_{i}^{S}\right| \leq\left|L_{j}^{\prime}-L_{i}^{A}\right|+\Delta_{j}^{\prime} .
$$

In the following, let $b_{i j k}$ be the difference in pseudoranges to $P_{i}^{A}$ between $V_{j}$ and $V_{k}$ (see Equation 6):

$$
b_{i j k}=R_{i j}^{A}-R_{i k}^{A}=\left|P_{j}-P_{i}^{A}\right|-\left|P_{k}-P_{i}^{A}\right| .
$$

Equally, we define $b_{i j k}^{\prime}$ as the difference of pseudoranges of the claimed satellite location $L_{i}^{A}$ and the spoofed victim locations $L_{j}^{\prime}$ and $L_{k}^{\prime}$ (see Figure 4):

$$
\begin{aligned}
b_{i j k}^{\prime} & =R_{i j}^{\prime}-R_{i k}^{\prime} \\
& =\left|L_{j}^{\prime}-L_{i}^{A}\right|-\left|L_{k}^{\prime}-L_{i}^{A}\right|+\Delta_{j}^{\prime}-\Delta_{k}^{\prime} .
\end{aligned}
$$

\subsection{Spoofing to One Location}

Result 1. One or more receivers $V_{j} \in \mathcal{V}$ can be spoofed to any one location $L^{\prime}$ using a single attacker antenna. Spoofing multiple receivers to the same location $L^{\prime}$ will generally lead to different time offsets $\delta_{j}^{\prime}$ at each victim.

The reason for this is that the time-differences of arrival of the individual satellite signals determine the location that each receiver will compute. If the spoofed signals are all sent from the same attacker antenna, all victims will obtain the same time-differences. A detailed proof is given in Appendix A, along with a discussion of the resulting time differences at the victims.

\subsection{Spoofing to Multiple Locations}

We next consider multiple receivers at distinct physical locations $P_{1}, \ldots, P_{n}$ that the attacker tries to spoof to the locations $L_{1}^{\prime}, \ldots, L_{n}^{\prime}$. Following Result 1 , an attacker can spoof any number of receivers in the transmission range to the same coordinates $L^{\prime}$ with differing $\delta_{j}^{\prime}$. If the victims have a way of establishing (coarse) relative distances, e. g., by estimating their respective distances visually, or can detect their mutual time offsets, they are able to detect such attacks. Therefore, we will now focus on attacks in which multiple victims are shifted to a set of new locations that preserve their mutual distances and mutual time offsets.

As stated in Result 1, if the attacker is using only one transmission antenna, any possible placement of this antenna will lead to two victims computing their location to the same coordinates $L^{\prime}$, with a small time synchronization error. Hence, the attacker cannot use only one antenna to shift the victims to different locations. We will now show that, using multiple antennas, the attacker can spoof two victims to any locations while preserving their mutual time offsets, with certain restrictions on the time offset in the case of military GPS receivers.

Result 2. Two receivers at the physical locations $P_{1} \neq P_{2}$ can be spoofed to the locations $L_{1}^{\prime} \neq L_{2}^{\prime}$ and time offsets $\delta_{1}^{\prime}, \delta_{2}^{\prime}$ if the attacker is free to choose any $P_{i}^{A}$ and $L_{i}^{A}$. For each $s_{i}^{A}$, the possible transmission locations $P_{i}^{A}$ lie on one half of a two-sheeted hyperboloid defined by $L_{1}^{\prime}, L_{2}^{\prime}, \delta_{1}^{\prime}, \delta_{2}^{\prime}, L_{i}^{A}$, and $P_{1}, P_{2}$.

In order to spoof $V_{1}, V_{2}$ to $L_{1}^{\prime}, L_{2}^{\prime}$ and $\Delta_{1}^{\prime}, \Delta_{2}^{\prime}$, the attacker must send each $s_{i}$ such that it arrives with the correct delay at the physical locations of the victims, i. e., $b_{i 12}=b_{i 12}^{\prime} \forall s_{i}$. As $b_{i j k}$ is defined by $P_{i}^{A}$ and, likewise, $b_{i j k}^{\prime}$ is defined by $L_{i}^{A}$, the attacker can always find combinations of $P_{i}^{A}$ and $L_{i}^{A}$ that yield the correct pseudorange (for attacks on civilian GPS). He can then use Equation 8 to find the appropriate $\delta_{i}^{A}$.

In the case of military GPS, the attacker cannot change the claimed placements of the satellites: $L_{i}^{A}=L_{i}^{S}$. Hence, $b_{i 12}^{\prime}$ is determined by the selection of $L_{1}^{\prime}, L_{2}^{\prime}$ and $\delta_{1}^{\prime}, \delta_{2}^{\prime}$. In this case, Equation 8 yields one hyperboloid for each $s_{i}^{A}$ with possible values of $P_{i}^{A}$ and $\delta_{i}^{A}$.

We demonstrate this by giving a simple example: the victims are located at $P_{1}=(1,0,0)$ and $P_{2}=(-1,0,0)$, the physical distance between the victims is $\left|P_{1}-P_{2}\right|=2$. The attacker wants to spoof the two victims to the locations $L_{1}^{\prime}=(0,0,0)$ and $L_{2}^{\prime}=$ 


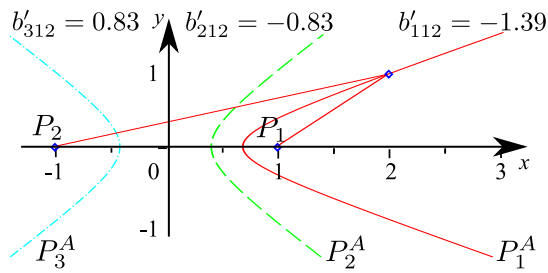

Figure 5: Hyperbolas of possible antenna placements for the attacker when impersonating a satellite for two victims (Example for Result 2, in 2D). Each hyperbola represents possible placements for an antenna $P_{i}^{A}$.

$(0,2,0)$, both with time offset zero: $\Delta_{1}^{\prime}=\Delta_{2}^{\prime}=0$. The attacker now (arbitrarily) chooses $L_{1}^{A}=(-3,-2,0), L_{2}^{A}=(-2,0,0)$, and $L_{3}^{A}=(-2,2,0)$ for the claimed satellite positions in the GPS messages. This determines three hyperboloids relative to $P_{1}$ and $P_{2}$ based on $b_{112}^{\prime}, b_{212}^{\prime}$, and $b_{312}^{\prime}$.

Result 3. A necessary condition for a successful GPS group spoofing attack is that $\forall V_{j}, V_{k}, \forall s_{i}, b_{i j k}^{\prime} \leq\left|P_{j}-P_{k}\right|$.

In other words, the difference $b_{i j k}^{\prime}$ of the perceived pseudoranges of each signal $s_{i}^{A}$ at any two spoofed victim locations $L_{j}^{\prime}$ and $L_{k}^{\prime}$ must be smaller than or equal to the distance between the victims' physical locations $P_{j}$ and $P_{k}$. From Equation 11 and the triangle inequality it follows that $b_{i j k} \leq\left|P_{j}-P_{k}\right|$. Since it must hold that $b_{i j k}^{\prime}=b_{i j k}$, if $b_{i j k}^{\prime}>\left|P_{j}-P_{k}\right|$ for any $s_{i}$, then there is no possible solution for the attacker's placement $P_{i}^{A}$. Thus we get

$$
\left|P_{j}-P_{k}\right| \geq\left|L_{j}^{\prime}-L_{i}^{A}\right|-\left|L_{k}^{\prime}-L_{i}^{A}\right|+\Delta_{j}^{\prime}-\Delta_{k}^{\prime}
$$

as a necessary condition for a successful attack.

As we know from Result 2, for two victims, all possible antenna placements for the attacker lie on a hyperboloid defined by $P_{j}, L_{j}^{\prime}, \delta_{j}^{\prime}$ and $L_{i}^{A}$. We will now extend this result to the case of three and more victims. In the following, we assume that $b_{i j k}^{\prime} \leq$ $\left|P_{j}-P_{k}\right|$ is fulfilled $\forall V_{j}, V_{k}$ and $\forall s_{i}$, i. e., it is physically possible to spoof the locations of the receivers.

Result 4. In a GPS group spoofing attack on three victims $V_{1}, V_{2}, V_{3}$ to specific locations $L_{j}^{\prime}$ and time offsets $\delta_{j}^{\prime}$, all possible attacker placements $P_{i}^{A}$ lie on the intersection of two hyperboloids defined by $b_{i 12}^{\prime}, b_{i 13}^{\prime}$.

This can be shown by constructing two hyperboloids using $b_{i 12}^{\prime}$ and $b_{i 13}^{\prime}$ as in Result 2. Both hyperboloids yield the possible placements of attacker's antennas to achieve the correct pseudorange for $V_{1}, V_{2}$ or $V_{1}, V_{3}$, respectively. Each point on the intersection of the two hyperboloids has a specific $\delta_{i}^{A}$ and is at the correct distance to all three victims. Therefore, all points of this space curve are valid $P_{i}^{A}$ to solve the group spoofing problem.

We can extend our example from Result 2 by a third victim placed at $P_{3}=(1,5,0)$, which is spoofed to $L_{3}^{\prime}=(1,1,0)$ with $\delta_{3}^{\prime}=0$. This reduces the possible locations from the hyperboloid as shown in Figure 6(a) to the intersection curve of the hyperboloids constructed using $b_{i 12}^{\prime}$ and $b_{i 13}^{\prime}$, as shown in Figure 6(b).

Result 5. In a GPS group spoofing attack on four victims $V_{1}, \ldots, V_{4}$ to specific locations $L_{j}^{\prime}$ and time offsets $\delta_{j}^{\prime}$, there are at most two possible placements for $P_{i}^{A}$ to impersonate a satellite at $L_{i}^{A}$. These are the intersection points of three hyperboloids defined by $b_{i 12}^{\prime}$, $b_{i 13}^{\prime}, b_{i 14}^{\prime}$.
As previously, to show this, we consider each signal $s_{i}^{A}$ separately. By computing $b_{i 12}^{\prime}, b_{i 13}^{\prime}, b_{i 14}^{\prime}$ (and $b_{i 11}^{\prime}=0$ ) according to Equation 11 and setting $b_{i j k}=b_{i j k}^{\prime}$, we can construct three hyperboloids. Their intersection points are possible placements for the antennas of the attacker. As the intersection of two hyperboloids yields a spaced curve, the intersection of three hyperboloids is an intersection of this curve with a third hyperboloid, which results in at most two points. We can also arrive at this number of solutions by considering the system of four quadratic equations based on Equation 7. These can be transformed into three linear and one quadratic equation [1], defining the solutions for the location $L_{i}^{A}$ and time offset $\delta_{i}^{A}$. As the quadratic equation has at most two solutions [1], and each of the linear equations has one unique solution, there are at most two solutions for the attacker's position and transmission time.

This result can also be observed in our example by adding a fourth victim placed at $P_{4}=(10,0,0)$, which is spoofed to $L_{4}^{\prime}=$ $(-1,0,0)$ with $\delta_{4}^{\prime}=0$. The possible placements for the attacker's antenna is now the intersection of the previously obtained curve with another hyperboloid, yielding two points only (Figure 6(c)).

Result 6. In a GPS group spoofing attack on five or more victims $V_{1}, \ldots, V_{n}$ to specific locations $L_{j}^{\prime}$ and time offsets $\delta_{j}^{\prime}$, there is at most one possible placement for $P_{i}^{A}$ to impersonate a satellite at $L_{i}^{A}$. This is the intersection point of $n-1$ hyperboloids defined by $b_{i 12}^{\prime}, \ldots, b_{i 1 n}^{\prime}$.

This result directly continues our previous reasoning: Each added victim adds another hyperboloid to the set of hyperboloids which must intersect to yield a possible $P_{i}^{A}$. For five or more receivers, the set of $(n-1)$ linear equations and one quadratic equation is overdetermined, and therefore has at most one solution.

From Result 5, we know that for military GPS receivers, there are at most two solutions for a given combination of $P_{j}, L_{j}^{\prime}, \delta_{j}^{\prime}$, and $L_{i}^{A}=L_{i}^{S}$. For attacks on civilian GPS receivers, the attacker can influence the position of the two solutions of the system of equations by changing the claimed satellite location $L_{i}^{A}$. We will now give an intuition where these solutions are located for a formationpreserving GPS spoofing attack.

Result 7. When spoofing a group of GPS receivers $V_{1}, \ldots, V_{n}$ such that the formation (i.e., the mutual distances and relative time offsets) is preserved, there is always at least one solution to the decisional group GPS spoofing problem.

One way to show this result is to use an affine transformation to describe the relation between physical and spoofed locations of the receivers and senders. If the formation of the victims is preserved, there exists a bijective affine augmented transformation matrix $T$ which describes this translation and rotation. Assuming that $L$ and $P$ are represented as augmented row vectors, we can therefore write $T \cdot L_{j}=L_{j}^{\prime}$. Then, the inverse transformation $T^{-1}$ applied to $L_{i}^{A}$ will yield a possible antenna placement $P_{i}^{A}=T^{-1} \cdot L_{i}^{A}$, because all pseudoranges $R_{i j}^{\prime}$ between $L_{j}^{\prime}$ and $L_{i}^{A}$ and the measured range $R_{i j}$ between $P_{i}^{A}$ and $P_{j}$ will be the same (the transformation preserves the Euclidean distance).

As a consequence of Results 6 and 7, spoofing five or more receivers while retaining their formation has exactly one solution, an affine transformation of the claimed satellite position $L_{i}^{A}$.

Summary of results: Table 2 gives an overview of sets of possible positions $P_{i}^{A}$ for the attacker's antenna depending on the number 


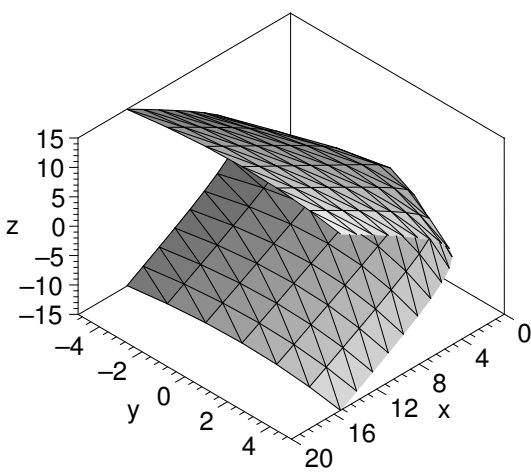

(a) 2 receivers

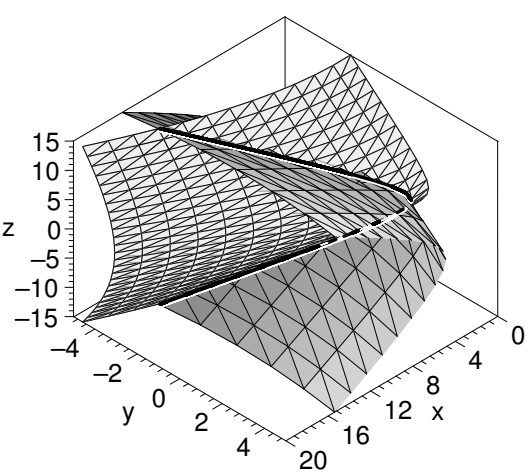

(b) 3 receivers

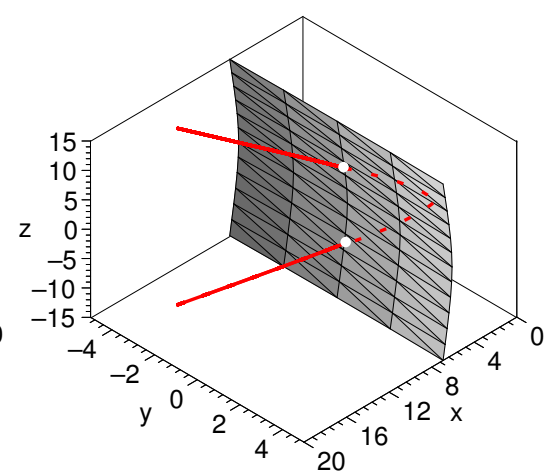

(c) 4 receivers

Figure 6: Visualization of possible attacker placements. For (a) two victims, all points on the hyperboloid are viable solutions; for (b) three victims the solutions lie on a curve (red/white intersection); and (c) for four victims only two points are viable solutions (white dots).

\begin{tabular}{|c|c|c|c|}
\hline \multirow[b]{2}{*}{ 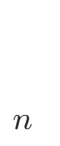 } & \multirow{2}{*}{$\begin{array}{c}\begin{array}{c}\text { Spoofing to } \\
\text { one location }\end{array} \\
\text { Civ. \& Mil. GPS }\end{array}$} & \multicolumn{2}{|c|}{$\begin{array}{c}\text { Spoofing to multiple } \\
\text { locations (preserved formation) }\end{array}$} \\
\hline & & Civilian GPS & Military GPS \\
\hline 1 & $P_{i}^{A} \in \mathbb{R}^{3}$ & - & - \\
\hline 2 & $P_{i}^{A} \in \mathbb{R}^{3}$ & set of hyperboloids & one hyperboloid \\
\hline 3 & $P_{i}^{A} \in \mathbb{R}^{3}$ & $\begin{array}{l}\text { set of intersections } \\
\text { of two hyperboloids }\end{array}$ & $\begin{array}{l}\text { intersection of } \\
\text { two hyperboloids }\end{array}$ \\
\hline 4 & $P_{i}^{A} \in \mathbb{R}^{3}$ & set of 2 points & 2 points \\
\hline$\geq 5$ & $P_{i}^{A} \in \mathbb{R}^{3}$ & set of points & 1 point \\
\hline
\end{tabular}

Table 2: Summary of results for the number of possible attacker locations $P_{i}^{A}$ for $n$ victims.

of victims and on the target locations: spoofing all receivers to one location or each victim to a different location with a preserved formation. The results are shown for civilian and military GPS; 'hyperboloid' refers to half of a two-sheeted hyperboloid. In the table we assume that the condition of Result 3 holds.

The results in Table 2 show that there are no restrictions on the attacker's position for spoofing any number of victims to one location $\left(P_{i}^{A} \in \mathbb{R}^{3}\right)$. With an increasing number of victims and a constant formation, the attacker is getting more and more restricted in terms of his antenna placement. For civilian GPS, the attacker has more degrees of freedom because he can select claimed (false) satellite locations $L_{i}^{A}$ and thus influence the hyperboloid, intersection of hyperboloids, etc., whereas these are fixed for military GPS (i. e., there is only one specific hyperboloid of attacker positions for each transmitted signal per pair of victims).

\section{EXPERIMENTS ON SATELLITE-LOCK TAKEOVER}

A GPS spoofing attack in the presence of legitimate GPS satellite signals requires the attacker to make the victim stop receiving signals from the legitimate satellites and start receiving the attacker's signals. If this takeover is noticed by the victim, e. g. because the victim suddenly loses contact to previously seen satellites, it can detect the spoofing attack. While the victim might lose contact due to random noise or environmental changes, the attacker ideally should take over without being noticed. We say that the receiver has a lock on a specific transmitter when it is already receiving data

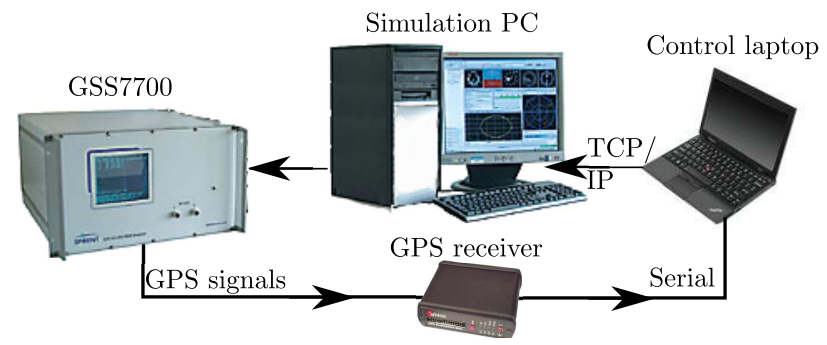

Figure 7: The experimental setup.

from that satellite. The satellite lock makes spoofing attacks harder since a spoofing signal is likely to be misaligned (in phase, Doppler shift, or data content) to the legitimate signal. When the attacker's signal is turned on, this momentary interruption in the data-flow from that satellite could cause the victim to be temporarily unable to compute his position. Therefore, we now investigate how the attacker can take over the victim's lock with the victim losing the ability to calculate its position, even for a moment.

In Section 3 we assumed a strong attacker, who is always able to generate signals with perfect timing and power level, and who has perfect knowledge of his own and the victim's position. In a practical attack, many of these assumptions might be invalid. We conduct experiments to evaluate the influence of such imperfections. Because we do not change the claimed location of the satellite in the data sent by the attacker, all discussed imperfections should apply equally for military and public GPS receivers.

\subsection{Experimental Setup and Procedure}

In our experiments, the spoofing signals and the legitimate GPS signals are sent over a cable to eliminate the influence of the transmission channel. This enables us to measure the unique influence of the parameters of interest while disregarding channel and antenna noise. These results therefore show the minimal precision of the signal parameters required for a successful attack on our target platform.

We conduct the lock takeover attacks using a Spirent GSS7700 GPS simulator (see Figure 7). The GPS signal simulator is a hardware device that generates GPS signals and is controlled by a dedicated simulation PC running the SimGen simulation software package [20]. The GSS7700 GPS simulator generates two independent 


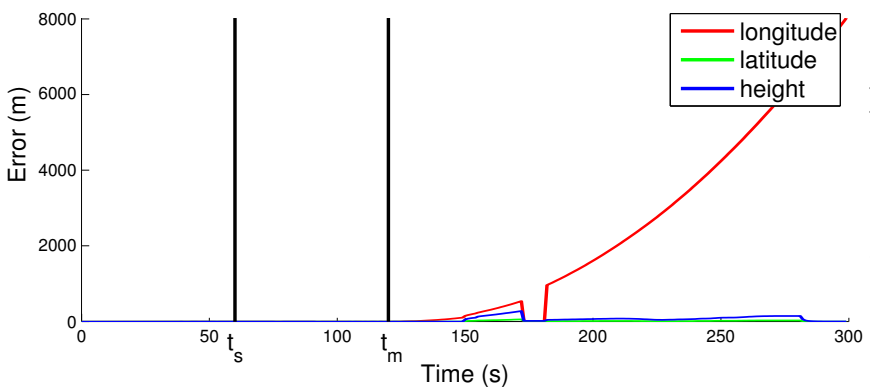

(a) Sample run with $+0 \mathrm{~dB}$ power offset

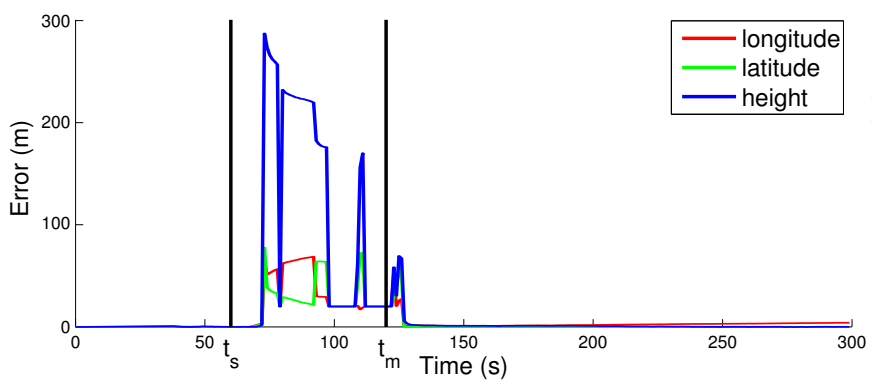

(c) Sample run with $120 \mathrm{~ns}$ time offset

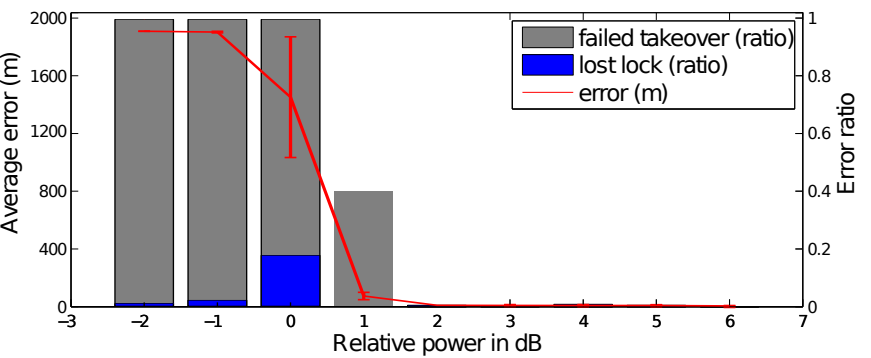

(b) Average error as a function of power offset

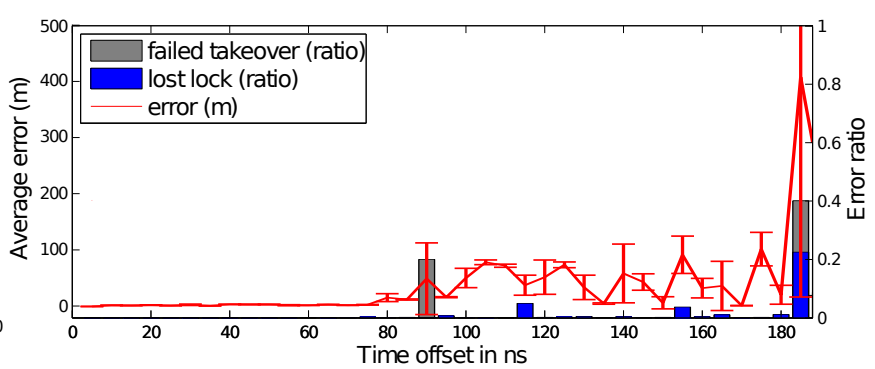

(d) Average error as a function of time offset

Figure 8: (a-b) Effects of relative signal power. (a) Example of unsuccessful takeover with too little power used. The spoofing signal is switched on at $t_{s}=60 \mathrm{~s}$ and starts moving at $t_{m}$. (b) Average error over the measurement as a function of relative power. (c-d) Example of effects of spoofing signals with time offset. (c) During the takeover, the location jumps, in particular the height. The spoofing signal is switched on at $t=60 \mathrm{~s}$. (d) Average error over the measurement as a function of the time offset.

GPS constellations with up to 16 satellites in each. One constellation is simulating the signals from the legitimate GPS satellites, and the other is simulating the attacker's signals. Both are mixed together and sent to the GPS receiver via a wired connection. The GPS receiver in our experiments is an Antaris evaluation kit by $\mathrm{u}-$ blox, containing the ATR0600 GPS chip from Atmel.

At the start of each experiment, we send only the legitimate GPS signals for a static location. We reset the GPS receiver to make sure all experiments are independent and no internal state is kept from a previous experiment. After about 30 seconds the GPS receiver will lock on to enough satellites to be able to calculate a stable position. This position is the legitimate position $L$ and the goal of the attacker is now to move the victim to a new location $L^{\prime}$ such that (i) the victim is continuously able to compute its position (ii) no noticeable discontinuities in the location are reported by the victim's receiver.

The attack then consists of two phases: first, the attacker sends signals which are supposed to match the legitimate satellites' signals at the location of the victim. These are generated by the attacker by approximating the current location of the victim as $L_{\text {init }}$, and constructing signals with time delays and data content appropriate for that location (see Section 4.1). This first phase lasts for one minute to allow the victim to lock on to the new signals. In the second phase, the attacker start to move the spoofed location towards the final location $L^{\prime}$, imitating an acceleration of $0.5 \mathrm{~m} / \mathrm{s}^{2}$. After 3 minutes, the final location is reached. If this final location is not remotely close to $L^{\prime}$ (height difference $\leq 150 \mathrm{~m}$, horizontal distance $\leq 1 \mathrm{~km}$ ), we consider the takeover failed.

We vary the distance between the victim's true location $L$ and its initial location as assumed by the attacker $L_{\text {init }}$ as one of the parameters in the experiments. We refer to this distance as the location offset $d_{\text {init }}=\left|L-L_{\text {init }}\right|$. The other parameters we investi- gate are relative signal power, relative time offset and constant time offset. For each parameter value, five experiments were run.

We say that the lock takeover was successful if at the end of the experiment the victim's final location is close to $L^{\prime}$. If the victim is close to $L^{\prime}$ but was close unable to compute a valid position for more than one second during the lock takeover, we consider the attack a partial success and use the number of seconds the victim was not able to calculate a valid position as an error metric.

\subsection{Results of the Experiments}

Relative signal power of the spoofing signal: In this experiment, ideal spoofing signals are sent, but the power of the spoofing signals is varied between $-2 \mathrm{~dB}$ and $+8 \mathrm{~dB}$ relative to the legitimate signals.

Figure 8(a) shows the effect of using spoofing signals that have the same power as the legitimate signals. In this figure, $t_{s}$ marks the time at which the spoofing signals are turned on and $t_{m}$ the time when the spoofed location starts to move away from $L_{i n i t}$. The errors in longitude, latitude, and height are shown separately and are measured between the location as reported by the receiver and the one sent by the simulator. Although the victim reports the spoofed location for some time, it switches back to $L$ after 170 s of the experiment, which causes the growing error in longitude.

Figure 8(b) shows the error in meters between the position reported by the GPS receiver and the location sent by the attacker, as a function of the relative power of the attacker's signals. The error bars show the standard deviation for the error value over the five experimental runs. The gray bars indicate the ratio of experiments in which the receiver was unable to determine its position during the experiment. We use this as a metric to evaluate the smoothness of the lock takeover. If the receiver reported a location too far away from $L^{\prime}$, we count this run as failed takeover. Blue bars in the figure 


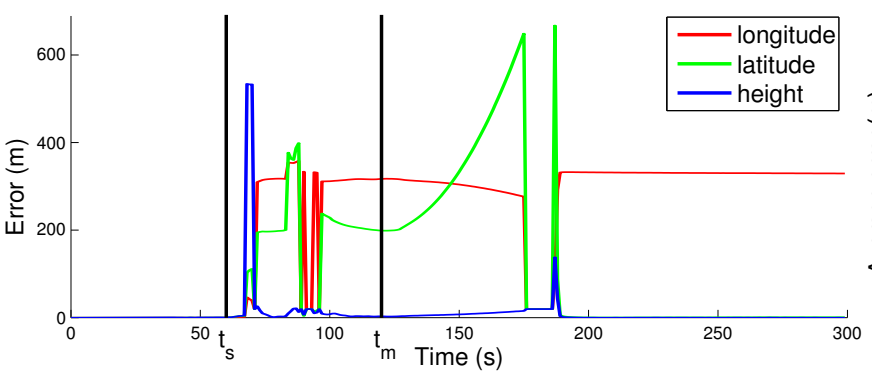

(a) Sample run with $340 \mathrm{~m}$ location offset

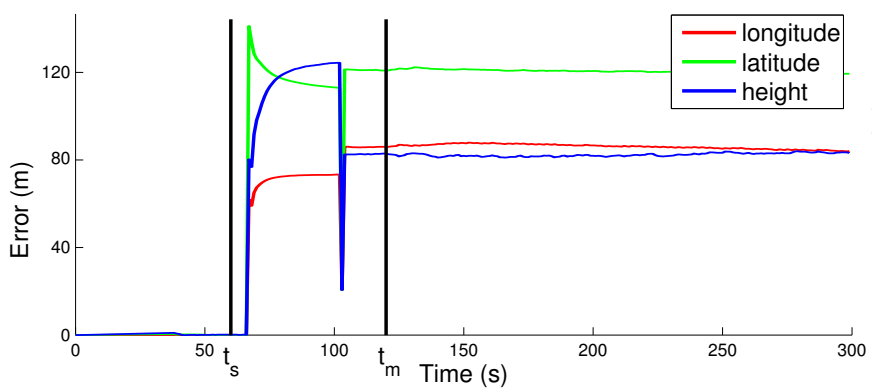

(c) Sample run with $140 \mathrm{~ns}$ time delay mismatch offset

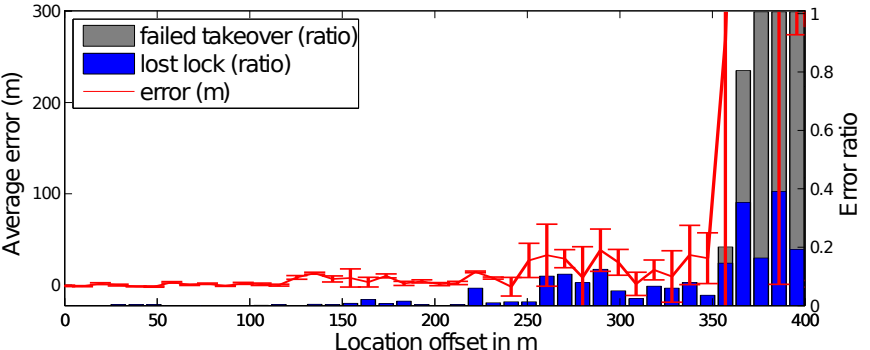

(b) Average error as a function of location offset

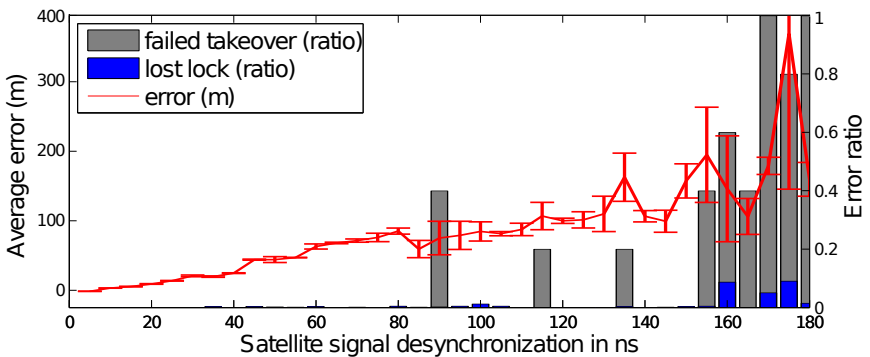

(d) Average error as a function of time delay mismatch

Figure 9: (a-b) Example of effects of spoofing signals with location offset. (a) Example with 340m offset. During the takeover, the location is unstable. The spoofing signal is switched on at $t=60 \mathrm{~s}$. (b) Average error over the measurement as a function of the location offset. (c-d) Example of effects of spoofing signals with inconsistent time offset for half of the satellites. (c) With a 140ns time offset between the attacker's satellites, the takeover leads to an unstable lock. The spoofing signal is switched on at $t=60 \mathrm{~s}$. (d) Average error over the measurement as a function of the time delay mismatch.

denote the ratio of attempts in which the GPS receiver was unable to compute a valid location.

It can be seen that for at least $2 \mathrm{~dB}$ more power, the receiver consistently locks onto the spoofing signals without any offset occurring. $2 \mathrm{~dB}$ of power is sufficiently low to not be detected by power based spoofing-countermeasures in practice.

Constant time offset influence: The second question we investigate is the effect of a general delay on all signals sent by the attacker relative to the legitimate signals. Such time delays can occur if the attacker's system setup is not perfectly compensating for internal delays, the distance to the victim is unknown or the system clock of the attacker is not synchronized perfectly to the clock of the legitimate GPS satellites. The interesting question is if such a general time offset will result in detectable errors in the victim's reported position, and if such a time offset will increase the chance of the victim losing lock completely during the takeover. To evaluate the influence of a constant time offset, we run the tests with time offsets between 0ns and 240ns. We plot the location error between the attacker's intended location and the actual location reported by the victim an example run in Figure 8(c). The effects are consistent over several runs with the same parameters, but can vary quite a lot with these parameters.

In Figure 8(d), we show the general relation between the average errors during the measurement as a function of the time offset for the first 120ns. After this time, lock takeover was not working consistently any more.

Location offset influence: In this series of experiments we determine the influence of an offset $d_{\text {init }}$ between the position of the victim as determined from the legitimate satellites $L$ and the spoofing signals sent by the attacker $L_{\text {init }}$. We evaluate the influence of such a location offset for values between 0 and $450 \mathrm{~m}$. Similarly to the time offset, this location offset can lead to a relatively large error during the lock takeover. An example with offset of $340 \mathrm{~m}$ is given in Figure 9(a).

In Figure 9(b), we show the average error as a function of the location offset. Regardless of the intermediate errors, eventually the victim always synchronizes to the attacker's signals in all our experiments. This shows that the initial position is not very sensitive to small errors. If an attacker knows the location of his victim to within about 100 meters, he can perform a smooth takeover without the victim losing lock. There will of course be a detectable jump in position from $L$ to $L_{\text {init }}$ when the attackers signal is turned on but the victim will not lose lock with any satellite.

Relative time offset influence: In the case where the attacker has access to more than one transmission antenna, he can send the spoofing signals using two or more omnidirectional antennas (see Section 4). Depending on the relative position of the individual antennas, the victim will receive the spoofing signals with different time delays. Relative time offsets of the signals can also be caused by inaccuracies in the delay setup in the case of military GPS signals. In this experiment, we evaluate the consequences of having half of the spoofed satellite signals shifted by a fixed amount of time relative to the other half of the signals. In Figure 9(c), we show an example run with a time delay mismatch of 140ns. The results for all tested values are presented in Figure 9(d).

\subsection{Discussion on Practical Issues in Spoofing Attacks}

Because our experiments are based on a single GPS receiver, we do not attempt to make precise general statements about the parameter values that are necessary to perform a seamless takeover of any platform. Instead we point out that ranges with acceptable 


\begin{tabular}{lc} 
& $\begin{array}{c}\text { Parameter value required } \\
\text { for successful spoofing }\end{array}$ \\
\hline Relative signal power & $\geq+2 \mathrm{~dB}$ \\
Constant time offset & $\leq 75 \mathrm{~ns}$ \\
Location offset & $\leq 500 \mathrm{~m}^{\dagger}$ \\
Relative time offset & $\leq 80 \mathrm{~ns}$ \\
\hline
\end{tabular}

Table 3: Required parameter ranges for seamless lock-takeover in a GPS spoofing attack in our experiments.

values exist and we present the values for our receiver in Table 3.

According to our experiments, the constant time offset is sensitive to variation and should be less than $75 \mathrm{~ns}$. Anything more than that will cause the GPS receiver to lose lock when the spoofing signal is turned on. A value of $75 \mathrm{~ns}$ roughly corresponds to a distance of $22.5 \mathrm{~m}$, meaning that the attacker must know the distance from himself to the victim with an accuracy of $22.5 \mathrm{~m}$ (or better) - a higher offset will cause the victim to lose lock due to the signal (chip phase) misalignment. We found that the initial location offset will cause a noticeable jump of the victim's reported position during the attack. Large offsets could therefore be detected by the victim by monitoring its position. Any change in the arrival time of the signal from different antennas will directly impact the position calculated by the victim. If the relative time offset gets above 80ns the signals are sufficiently misaligned to cause the receiver to lose lock. This means that, if an attacker has multiple antennas, he must precisely know the distance from each antenna to the attacker in order to be able to spoof a desired location.

\section{GPS SPOOFING COUNTERMEASURE}

Spoofing detection based on lock loss has two disadvantages: (i) strong attackers can achieve a seamless satellite-lock takeover, and (ii) lock loss can occur due to natural causes (e.g. signal loss in a tunnel). We propose a countermeasure against GPS spoofing attacks that does not rely on the signal analysis or on the lock loss of signal. Instead, our mechanism is based on our insights of Section 4 and relies on the use of several GPS receivers. These GPS receivers can be deployed in a static, known formation, e.g., they are fixed on the deck of a cargo ship (see Figure 10). The basic idea of the countermeasure is the following: If the GPS receivers can exchange their individual GPS locations, they can check if their calculated locations preserve their physical formation (within certain error bounds). In the case that the calculated GPS locations do not match the known formation, an attack must be suspected and there should be a warning message. For the exchange of positioning information, the victim could also resort to wired connections if available (which would be resistant against spoofing and jamming attacks).

Even if only two GPS receivers are used, this countermeasure can detect any attacker that is only using a single antenna. As shown in Result 1, in case of a single-antenna attack both GPS receivers would report the same location (with small time offsets).

As shown in Results 4-6, a strong attacker using multiple antennas could attempt to send signals such that the mutual distances between multiple receivers are preserved. Nevertheless, each additional receiver of the victim makes these spoofing attacks exceedingly more difficult because the space of possible antenna placements for the attacker gets reduced significantly (see Table 2). From Results 6 and 7 we know that there exists only one location per satellite where the attacker can place his antenna; this location is the rotated and translated satellite position of the GPS signal. Con-

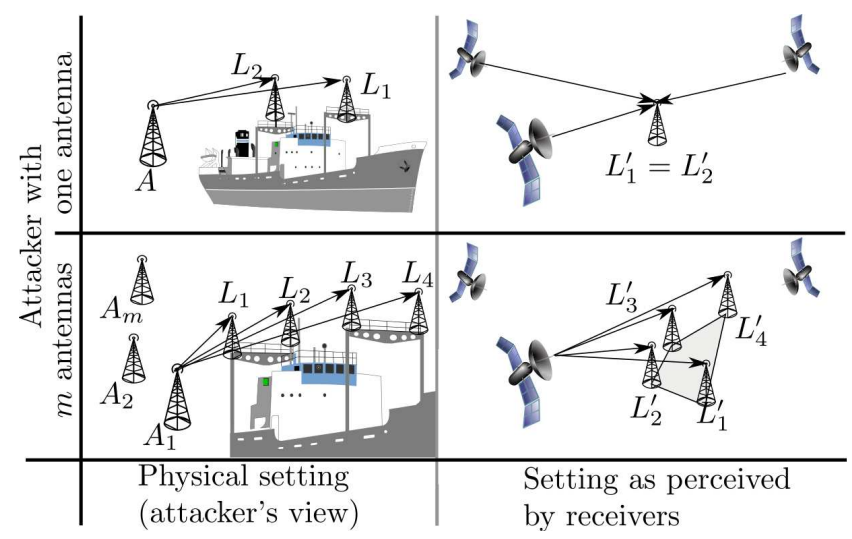

Figure 10: Proposed countermeasures: For an attacker with a single antenna, the two-receiver countermeasure is enough. If the attacker uses multiple antennas, four (or more) receivers severely restrict the attacker's antenna placements. Wrong antenna placements will change the distances of the receivers and can thus be detected.

ducting such an attack is very difficult. It becomes even impossible if the victim can hide the exact positioning of at least one GPS receiver from the attacker (e. g., by keeping it mobile on the vehicle) such that the attacker cannot adapt to its position.

In summary, our countermeasure requires no modifications of the GPS signal, the satellite infrastructure, or the GPS receiver, it is resistant against a wide range of attackers, and it can be deployed using multiple standard GPS receivers.

Outlook: Further possible applications are not restricted to mobile scenarios with a fixed formation (such as in the cargo ship example above). The countermeasure can also be applied (i) to fixed and static (i. e., immobile) settings where GPS is used for time synchronization and (ii) to mobile settings with varying formations (e.g., mobile formation of cars, robots, etc.). In the latter case, the devices can apply additional ranging techniques to identify their formation and use it in the sanity check with the calculated GPS locations (as long as the ranging techniques are secure [?,2, 6, 10, 18]). We leave the elaboration of these ideas for future work.

\section{CONCLUSION}

In this paper, we analyzed the requirements for successful GPS spoofing attacks on individuals and groups of victims with civilian or military GPS receivers. In particular, we identified from which locations and with which precision the attacker needs to generate its signals in order to successfully spoof the receivers.

For example, we show how spoofing a group of victims can only be achieved from a restricted set of locations, if the attacker aims to preserve the mutual distances and time offsets of the victims. With growing size of the group of victims, less spoofing location become available, until only single points remain for 5 victims or more. In addition, we discussed the practical aspects of seamless satellitelock takeover. We used a GPS signal generator to perform a set of experiments in which we investigated the required precision of the attacker's spoofing signals. Besides demonstrating the effects of such lock takeovers on the victim, our results include minimal bounds for critical parameters to allow a seamless takeover of our target platform. Finally, we proposed a technique for the detection of spoofing based on a group of standard GPS receivers (without specific spoofing detection measures) in a static formation. 


\section{REFERENCES}

[1] Bensky, A. Wireless Positioning Technologies and Applications. GNSS Technology and Applications Series. Artech House, 2008.

[2] Brands, S., AND Chaum, D. Distance-bounding protocols. In Workshop on the theory and application of cryptographic techniques on Advances in cryptology (EUROCRYPT) (1994), Springer.

[3] Cavaleri, A., Motella, B., Pini, M., and Fantino, M. Detection of spoofed GPS signals at code and carrier tracking level. In Proceedings of the 5th ESA Workshop on Satellite Navigation Technologies and European Workshop on GNSS Signals and Signal Processing (Navitec) (2010).

[4] Dolev, D., ANd YaO, A. C. On the security of public key protocols. IEEE Transactions on Information Theory 29, 2 (1983).

[5] ETTUS. Universal software radio peripheral (USRP). http://www.ettus.com.

[6] Hancke, G. P., And Kuhn, M. G. An RFID Distance Bounding Protocol. IEEE Computer Society.

[7] Humphreys, T. E., Ledvina, B. M., Psiaki, M. L., O'Hanlon, B. W., AND Kintner, P. M. Assessing the spoofing threat: Development of a portable GPS civilian spoofer. In Proceedings of the ION GNSS International Technical Meeting of the Satellite Division (2008).

[8] John A. Volpe National Transportation Systems CENTER. Vulnerability assessment of the transportation infrastructure relying on the global positioning system. Final Report, 2001.

[9] Johnston, R. G., And Warner, J. S. Think GPS cargo tracking $=$ high security . Think again. In Proceedings of Transport Security World (2003).

[10] Kuhn, M., Luecken, H., And Tippenhauer, N. O. UWB impulse radio based distance bounding. In Proceedings of the Workshop on Positioning, Navigation and Communication (WPNC) (2010).

[11] Kunn, M. G. An asymmetric security mechanism for navigation signals. In Proceedings of the Information Hiding Workshop (2004).

[12] Ledvina, B. M., Bencze, W. J., Galusha, B., And MILLER, I. An in-line anti-spoofing device for legacy civil GPS receivers. In Proceedings of the ION International Technical Meeting (2010).

[13] Montgomery, P. Y., Humphreys, T. E., And Ledvina, B. M. Receiver-autonomous spoofing detection: Experimental results of a multi-antenna receiver defense against a portable civil GPS spoofer. In Proceedings of the ION International Technical Meeting (2009).

[14] Motella, B., Pini, M., Fantino, M., Mulassano, P., Nicola, M., Fortuny-Guasch, J., WildemeERsCh, M., AND SYMEONIDIS, D. Performance assessment of low cost GPS receivers under civilian spoofing attacks. In Proceedings of the 5th ESA Workshop on Satellite Navigation Technologies and European Workshop on GNSS Signals and Signal Processing (Navitec) (2010).

[15] Navigation Center, U.S. Department of Home SECURITY. Global Positioning System, Standard Positioning Service: Signal Specification. http://www.navcen.uscg.gov, June 1995. 2nd edition.

[16] Papadimitratos, P., And Jovanovic, A. GNSS-based Positioning: Attacks and countermeasures. In Proceedings of the IEEE Military Communications Conference (MILCOM)
(2008)

[17] Papadimitratos, P., And Jovanovic, A. Protection and fundamental vulnerability of GNSS. In Proceedings of the International Workshop on Satellite and Space Communications (2008).

[18] Rasmussen, K. B., AND ČAPKUn, S. Realization of $\mathrm{rf}$ distance bounding. In Proceedings of the USENIX Security Symposium (2010).

[19] ScotT, L. Anti-spoofing \& authenticated signal architectures for civil navigation systems. In Proceedings of the ION GNSS International Technical Meeting of the Satellite Division (2003).

[20] Spirent Communications PlC. SimGEN simulation software. http://www.spirent.com.

[21] U. S. Departement of Defense. Global positioning system. standard positioning service. performance standard, Sep 2008.

[22] U. S. Government. Global positioning system. http://www.gps.gov, 2010.

[23] Warner, J. S., And Johnston, R. G. A simple demonstration that the global positioning system (GPS) is vulnerable to spoofing. Journal of Security Administration (2002).

[24] Warner, J. S., And Johnston, R. G. GPS spoofing countermeasures. Homeland Security Journal (2003).

\section{APPENDIX}

\section{A. PROOF OF RESULT 1}

To show Result 1, we first focus on a single receiver $V_{1}$ and civilian GPS. The attacker selects a target location $L^{\prime}$, a target time offset $\delta_{1}^{\prime}$, and any arbitrary attacker location $P_{i}^{A}$. Given this, Equation 8 yields $\Delta_{i}^{A}$. Using one transmission antenna (i.e. $P_{1}^{A}=$ $\left.P_{j}^{A} \forall j\right)^{5}$, the attacker transmits all signals $s_{i}^{A}$ with the delay $\delta_{i}^{A}=$ $\Delta_{i}^{A} / c$.

While this will successfully spoof the location and time of one victim, other victims in the vicinity will receive the same signals with slight time delay or advancement. We now consider a set of receivers $\mathcal{V}=\left\{V_{1}, \ldots, V_{n}\right\}$ that are positioned at different physical locations $\mathcal{P}=\left\{P_{1}, \ldots, P_{n}\right\}$.

Since the attacker sends all signals $s_{i}^{A}$ from the same position $P_{1}^{A}=P_{2}^{A}=\ldots$, we can follow that $b_{1 j k}=b_{2 j k}=\ldots$ for all signals $s_{i}^{A}$. To compute the effect of the offset on the pseudoranges on each victim, we can express each victims' pseudorange relative to the pseudorange of the first victim: $R_{i j}=R_{i 1}+b_{1 j 1}$. Each victim will measure pseudoranges based on their physical distances to the attacker: $R_{i j}^{\prime}=R_{i j}^{A}$. We can now substitute (11) into (7) and get the following equation for each signal $s_{i}^{A}$ and $V_{j}$ :

$$
\left|L_{j}^{\prime}-L_{i}^{A}\right|=R_{i 1}^{\prime}-\left(\Delta_{j}^{\prime}-b_{1 j 1}\right) .
$$

Thus, for every $V_{j}$, these equations only differ by the different value $\left(\Delta_{j}^{\prime}-b_{1 j 1}\right)=\Delta_{1}^{\prime}$. This means that all $V_{j}$ compute an identical location $L^{\prime}$, but different clock offsets $\delta_{j}^{\prime}$ :

$$
\delta_{j}^{\prime}=\delta_{1}^{\prime}+\frac{1}{c}\left(\left|P_{j}-P_{i}^{A}\right|-\left|P_{1}-P_{i}^{A}\right|\right) .
$$

\footnotetext{
${ }^{5}$ For civilian GPS, one physical transmission location for all attacker signals does not imply that the claimed locations $L_{i}^{A}$ in the spoofed messages are the same. For the victim to be able to compute its location, it must hold that $L_{1}^{A} \neq L_{2}^{A} \neq \ldots$.
} 
Result 1 shows that an attacker can make a group of victims believe to be at a specific location by sending one set of satellite signals from the same antenna. All victims will believe to be at the same location $L^{\prime}$, but with different time offsets. The additional time offset $\delta_{j}^{\prime}-\delta_{k}^{\prime}$ between victim $V_{j}$ and $V_{k}$ introduced by the attacker is bounded by their mutual distance $\left|\delta_{j}^{\prime}-\delta_{k}^{\prime}\right| \leq \frac{\left|L_{j}-L_{k}\right|}{c}$ and is typically on the order of nanoseconds for victims a few meters apart.

In attacks on military GPS, Equation 10 shows an interesting relation between the resulting time offset of the main victim $\delta_{1}^{\prime}$ and the distance between the spoofed location and each satellite: If $L_{1}^{\prime}$ is chosen such that $\left|L_{1}^{\prime}-L_{i}^{S}\right| \leq\left|P_{1}-L_{i}^{S}\right|$ for any $S_{i}$, then the time offset $\delta_{1}^{\prime}$ at the victim must be positive. On the other hand, since $\delta_{1}^{\prime}$ is the same for all satellites, only if the distances from $L_{1}^{\prime}$ to all satellites are enlarged (i. e., if $\left|L_{1}^{\prime}-L_{i}^{S}\right|>\left|P_{1}-L_{i}^{S}\right| \forall S_{i}$ ), the time offset of the victim can be made negative (causing the victim to advance its clock). The minimal value of $\delta_{1}^{\prime}$ is determined by

$$
\Delta_{j}^{\prime} \geq \max _{i}\left(\left|P_{1}-L_{i}^{S}\right|-\left|L_{1}^{\prime}-L_{i}^{S}\right|\right) \text {. }
$$

As the attacker can always delay the signals, he can arbitrarily delay the victims clock also in military GPS.

One direct conclusion for military GPS is that it is not possible to advance the victim's clock while retaining the original location $L_{1}^{\prime}=L_{1}$. The clock offsets of other victims $V_{2}, \ldots, V_{n}$ relative to the first victim as expressed in Equation 15 remain the same for attacks on military GPS if all signals are sent from the same location $P_{1}^{A}=P_{2}^{A}=\ldots$. 